\title{
UV Raman Studies of Peptide Conformation Demonstrate That Betanova Does Not Cooperatively Unfold ${ }^{\dagger}$
}

\author{
Mary N. Boyden and Sanford A. Asher* \\ Department of Chemistry, Chevron Science Center, University of Pittsburgh, Pittsburgh, Pennsylvania 15260 \\ Received July 19, 2001; Revised Manuscript Received September 5, 2001
}

\begin{abstract}
We used UV resonance Raman spectroscopy (UVRR) excited within the peptide bond $\pi \rightarrow$ $\pi^{*}$ electronic transitions and within the aromatic amino acid $\pi \rightarrow \pi^{*}$ electronic transitions to examine the temperature dependence of the solution conformation of betanova, a 20 -residue $\beta$-sheet polypeptide [Kortemme, T., Ramirez-Alvarado, M., and Serrano, L. (1998) Science 281, 253-256]. The $206.5 \mathrm{~nm}$ excited UVRR enhances the amide vibrations and demonstrates that betanova has a predominantly $\beta$-sheet structure between 5 and $82{ }^{\circ} \mathrm{C}$. The $229 \mathrm{~nm}$ excited UVRR, which probes the tyrosine and tryptophan side chain vibrations, shows an increase in the solvent exposure of the tryptophan side chains as the temperature is increased. Our results are consistent with the existence of an intermediate state similar to that calculated by Bursulaya and Brooks [Bursulaya, B. D., and Brooks, C. L. (1999) J. Am. Chem. Soc. 121, 9947-9951] and exclude the previously proposed two-state cooperative folding mechanism. Betanova's structure appears to be molten globule over the $3-82{ }^{\circ} \mathrm{C}$ temperature range of our study.
\end{abstract}

The completion of the human genome sequence makes available the primary sequence of all proteins synthesized by the human body. This represents only the first stage in the development of an understanding of the protein structural basis of disease. The next stage in this development requires an ability to predict the structure and function of proteins from their primary sequences. At present this is impossible, since not enough is known about the dynamics and thermodynamics of protein folding $(1-5)$.

The understanding of protein folding mechanisms is advancing as experimentalists develop better strategies for the de novo design of model peptides and better methodologies for characterizing equilibrium secondary structures, as well as the dynamics of folding. To date, synthetic schemes have been successfully developed for the synthesis of $\alpha$-helix-forming peptides, and much has been uncovered about their folding dynamics $(6-13)$. In contrast, only limited progress has been made toward understanding the synthesis of and the dynamics of $\beta$-sheet peptides. Primarily, studies have focused on $\beta$-hairpins rather than complete sheet structures $(14-18)$. Successful synthesis of three-stranded $\beta$-sheet structures has been accomplished only recently. However, these model peptides only form complete $\beta$-sheet structures under limited conditions, in the presence of organic solvents and with the use of unnatural amino acids (1922). Thus, there remains much to learn about $\beta$-sheet folding.

Significant advances have also occurred in the theoretical understanding of protein folding (23-28). Such work includes molecular dynamics simulations of energy landscapes that describe folding thermodynamics. Currently, some of these models accurately predict experimental results.

\footnotetext{
$\dagger$ We gratefully acknowledge support from NIH Grant R01GM30741.

* To whom correspondence should be addressed. Phone: (412) 6248570. Fax: (412) 624-0588. E-mail: asher+@pitt.edu.
}

Thus, theory can now impact how experimental data are interpreted. It seems likely that the most powerful insights into the rules of protein folding will result from combined experimental and theoretical studies of small peptides (29, 30).

In this study we used UV resonance Raman spectroscopy $(\mathrm{UVRR})^{1}$ to probe the temperature dependence of the structure of a small $\beta$-sheet peptide called betanova. Betanova is the first small peptide composed of naturally occurring amino acids to spontaneously form a three-stranded $\beta$-sheet structure in $100 \%$ water. This 20 residue (RGWSVQNGKYTNNGKTTEGR) peptide forms an antiparallel structure with a hydrophobic cluster on one face. The original experimental studies of betanova (31) appear to indicate that this $\beta$-sheet structure melts cooperatively to form a random coil structure as the temperature increases. In contrast, recent theoretical studies by Bursulaya and Brooks (32) found an energy landscape, which requires that the $\beta$-sheet should not melt cooperatively and should display an intermediate compact structure. Only a small barrier was found between the native state and a basin of compact intermediate structures, which had a $\beta$-sheet-like conformation but loosened side chain packing.

\section{EXPERIMENTAL PROCEDURES}

The Peptide Facility of the Pittsburgh Cancer Institute synthesized and purified betanova. For the UVRR experi-

\footnotetext{
${ }^{1}$ Abbreviations: UVRR, ultraviolet resonance Raman spectroscopy; $\mathrm{R}$, arginine; G, glycine; W and Trp, tryptophan; S, serine; V, valine; $\mathrm{Q}$, glutamine; $\mathrm{N}$, asparagine; $\mathrm{K}$, lysine; $\mathrm{Y}$ and $\mathrm{Tyr}$, tyrosine; $\mathrm{T}$, threonine; E, glutamic acid; PSSRS, pure secondary structure Raman spectra; CD, circular dichroism; NMR, nuclear magnetic resonance; AmI, amide I normal mode; AmII, amide II normal mode; AmIII, amide III normal mode; $\mathrm{C}_{\alpha}-\mathrm{H},(\mathrm{C}) \mathrm{C}_{\alpha}-\mathrm{H}$ bending normal mode; $\mathrm{Ar}$ str, aromatic ring stretching band.
} 


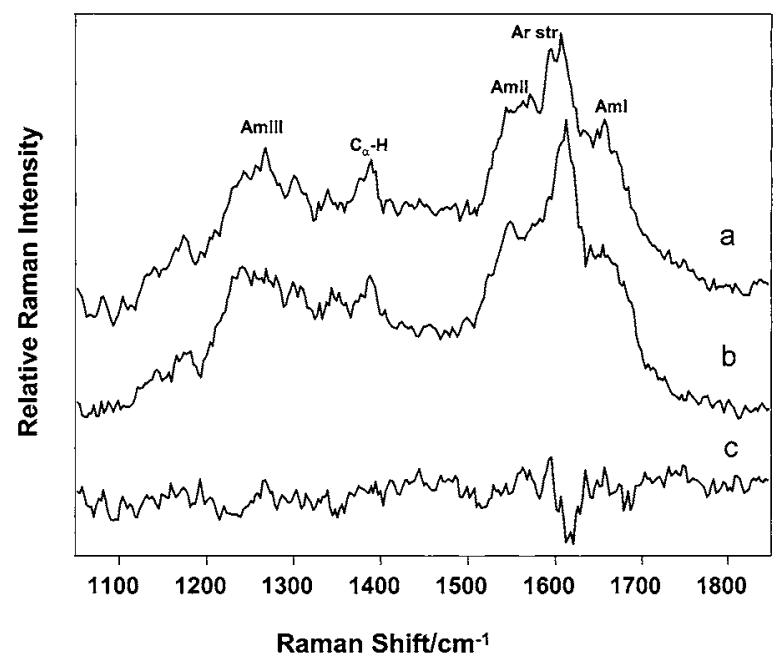

FIGURE 1: (a) $206.5 \mathrm{~nm}$ UVRR spectrum of betanova at $5{ }^{\circ} \mathrm{C}$. (b) $206.5 \mathrm{~nm}$ UVRR spectrum of betanova at $82{ }^{\circ} \mathrm{C}$. (c) Difference UVRR spectrum of betanova $\left(5^{\circ} \mathrm{C}-82{ }^{\circ} \mathrm{C}\right)$.

ments, samples were prepared by dissolving the peptide to a concentration of $0.13-0.30 \mathrm{mM}$ in a $5 \mathrm{mM}$ sodium acetate (EM Science, Gibbstown, NJ) buffer. Approximately $20 \mathrm{~mL}$ of the solution was circulated through a temperaturecontrolled quartz cuvette (Uvonics, Plainview, NY). Trytophan and tyrosine (Sigma Chemical Co., St. Louis, MO) were dissolved to concentrations of $\sim 1.5 \mathrm{mM}$ in $0.20 \mathrm{M}$ sodium perchlorate (Aldrich Chemical Co., Milwaukee WI). Intracavity frequency doubled krypton and argon ion lasers were used to produce 206.5 and $229 \mathrm{~nm}$ excitation. Raman scattering was collected using a $135^{\circ}$ backscattering geometry by using instrumentation that is described in detail elsewhere (33).

Quantitative determination of secondary structure composition was performed using the PSSRS methodology recently developed by Chi et al. (34). This technique models the experimentally obtained spectra by least-squares fitting of the basis spectra for the $\alpha$-helix, $\beta$-sheet, and random coil secondary structures to the measured peptide spectra. The accuracy of this technique was demonstrated by comparison of PSSRS results to X-ray crystallographic data of wellcharacterized proteins, and the relative standard deviation was 0.082 .

Circular dichroism (CD) spectra were measured by using a Jasco J-710 spectropolarimeter. Betanova was dissolved in $5 \mathrm{mM}$ sodium acetate to a concentration of $158 \mu \mathrm{M}$, and the spectra were measured using a temperature-controlled cell with a $1 \mathrm{~mm}$ path length.

\section{RESULTS AND DISCUSSION}

The $206.5 \mathrm{~nm}$ UVRR spectra of betanova are examined since they are dominated by amide bands, whose frequencies and intensities are sensitive to secondary structure. Figure 1a,b shows the $206.5 \mathrm{~nm}$ UVRR spectra of betanova at 5 and $82{ }^{\circ} \mathrm{C}$, respectively. The difference spectrum between the hot and cold samples is shown in Figure 1c. The UVRR of betanova is dominated by the AmI band at $1653 \mathrm{~cm}^{-1}$, the aromatic amino acid ring stretching band at $\sim 1610 \mathrm{~cm}^{-1}$, the AmII band at $1555 \mathrm{~cm}^{-1}$, and the AmIII band at 1242 $\mathrm{cm}^{-1}$ (Figure 1a,b). Also, there is a $\mathrm{C}_{\alpha}-\mathrm{H}$ bending band at $1384 \mathrm{~cm}^{-1}$, which is weak relative to the AmIII band. We

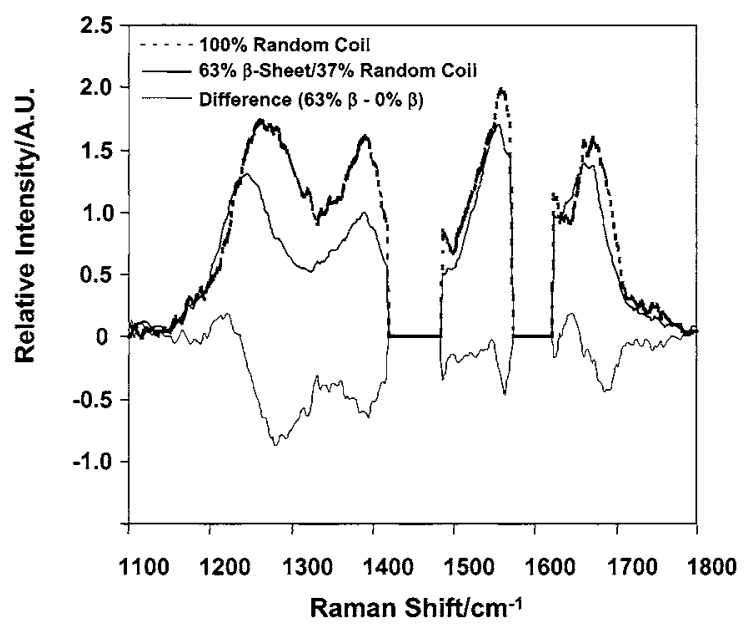

FIGURE 2: Illustration of the expected results for a two-state melting from the native ( $63 \% \beta$-sheet) to the unfolded ( $100 \%$ random coil). PSSRS modeled spectra depict a $63 \% \beta$-sheet polypeptide spectrum, a $100 \%$ random coil polypeptide spectrum, and their difference spectrum.

used the PSSRS methodology (34) to calculate betanova's secondary structure from its $206.5 \mathrm{~nm}$ excited UVRR spectrum.

Our best fit suggests a $63 \% \beta$-sheet content, close to that expected from the NMR data of Kortemme et al. (31), which shows that $\beta$-sheet structure begins at the $\mathrm{N}-\mathrm{H}$ of residue 3 and ends at the carbonyl of residue 17 . However, the fitting results clearly indicate that, although the peptide spectrum resembles that of a $\beta$-sheet spectrum, there are significant differences that cannot be modeled with our existing protein $\beta$-sheet, $\alpha$-helix, and random coil basis spectra.

Figure 1c shows the difference between the 5 and $82{ }^{\circ} \mathrm{C}$ UVRR spectra. Little or no changes occur for the amide and the $\mathrm{C}_{\alpha}-\mathrm{H}$ bending bands as betanova is heated from 5 to 82 ${ }^{\circ} \mathrm{C}$. The only temperature-induced spectral change occurs for the Tyr and Trp aromatic ring stretching band at $\sim 1610$ $\mathrm{cm}^{-1}$. The nearly flat difference spectrum indicates that there is little temperature dependence of the Ramachandran $\Phi$ and $\Psi$ angles. If there was a two-state melting of $\beta$-sheet to random coil, we would expect an increase in the frequency of the AmIII band to $\sim 1267 \mathrm{~cm}^{-1}$, an increase in the relative intensity of the $\mathrm{C}_{\alpha}-\mathrm{H}$ bending band, and a shifting of the AmI and AmII bands to higher frequencies. These expectations are derived from the Figure 2 spectral modeling (34).

Figure 2 models the UVRR spectra of two polypeptides, one of which has the $63 \% \beta$-sheet $/ 37 \%$ random coil conformations of betanova while the other has a $100 \%$ random coil structure. The difference spectrum models the spectral changes that should be observed upon melting of betanova to its random coil form. Specifically, there would be an increase in frequency for the AmI $\left(\sim 10 \mathrm{~cm}^{-1}\right)$, AmII $\left(\sim 10 \mathrm{~cm}^{-1}\right)$, and AmIII $\left(\sim 25 \mathrm{~cm}^{-1}\right)$ bands. In addition, we expect significant intensity increases for the AmIII and $\mathrm{C}_{\alpha}-\mathrm{H}$ bands, as well as more modest increases for the AmI and AmII band intensities. Furthermore, we anticipate an increase in the ratio of the $\mathrm{C}_{\alpha}-\mathrm{H} / \mathrm{AmIII}$ peak heights. Because we do not observe these spectral changes, we conclude that little change occurs in the betanova secondary structure as the temperature is increased from 5 to $82{ }^{\circ} \mathrm{C}$.

We attempted to unfold betanova to achieve the random coil form by acidifying the solution. However, betanova 


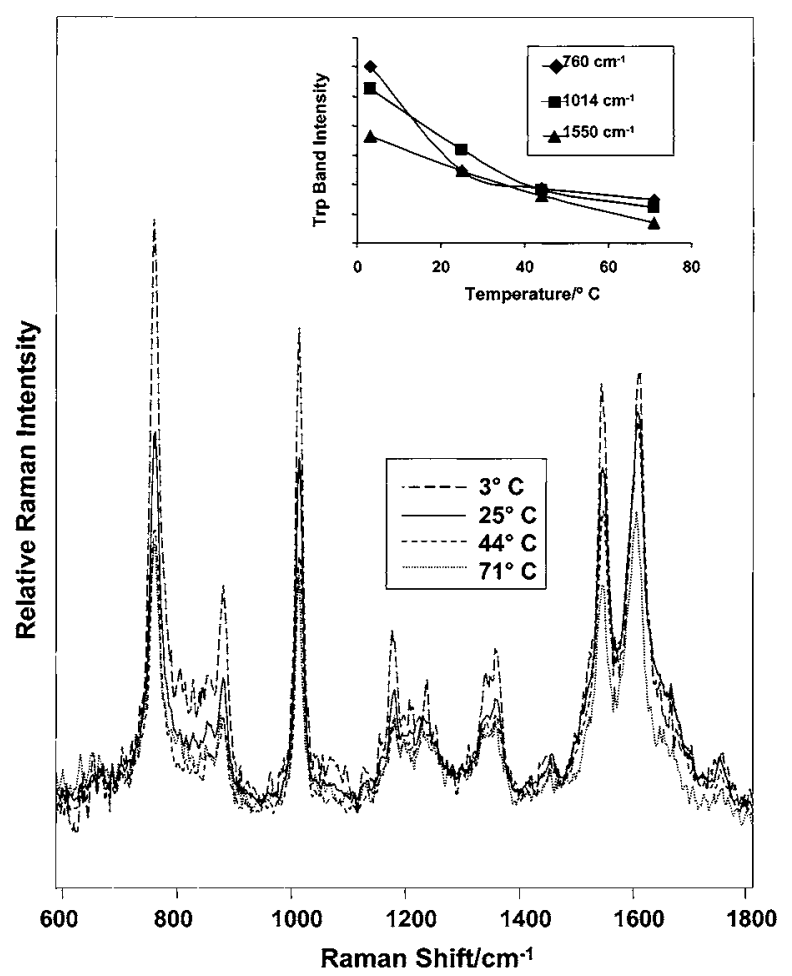

FIGURE 3: $229 \mathrm{~nm}$ excited UVRR of betanova at 3, 25, 44, and 71 ${ }^{\circ} \mathrm{C}$. The dependence of the tryptophan peak intensities on temperature is displayed in the inset.

became insoluble and precipitated from solution. We did not attempt the use urea because of its strong UV absorption.

For betanova, the only temperature-induced $206.5 \mathrm{~nm}$ excited Raman spectral change occurs for the $\sim 1610 \mathrm{~cm}^{-1}$ aromatic ring stretching band (Figure 1c). To obtain additional information on the peptide structural changes, we studied the aromatic amino acid spectral changes by using $229 \mathrm{~nm}$ excitation (Figure 3) to selectively probe the Tyr $\left(1180,1207\right.$, and $\left.1610 \mathrm{~cm}^{-1}\right)$ and Trp residues $(760,1014$, 1344,1362 , and $\left.1550 \mathrm{~cm}^{-1}\right)$. A temperature increase from 3 to $71{ }^{\circ} \mathrm{C}$ significantly decreases both the Tyr and the Trp band intensities. In the case of the $1610 \mathrm{~cm}^{-1}$ Tyr peak, this temperature increase results in the maximum peak intensity decreasing by $32 \%$. Over the same temperature range, an even more pronounced decrease of the Trp band intensities occurs; the peak height of the $1550 \mathrm{~cm}^{-1}$ band decreases by $48 \%$.

These betanova Tyr band intensity decreases do not signal a change in the Tyr environment because the changes are similar to those observed for Tyr in aqueous solution (Figure 4). These Raman intensity changes appear to result from a temperature-induced molar absorptivity decrease of the resonant $220 \mathrm{~nm}$ Tyr absorption band.

In contrast, the Trp intensity changes signal a change in environment. Trp monomer in solution does not show a significant temperature dependence for its $229 \mathrm{~nm}$ UVRR band intensities (Figure 4). Chi et al. (35) previously demonstrated that $229 \mathrm{~nm}$ excited Trp band intensities decrease in proteins upon unfolding, due to exposure of the Trp ring to water. This intensity decrease results from a blue shift of the Trp absorption band that results from water exposure.

The Trp intensity decrease observed for betanova probably results from an increased solvent exposure of the Trp side

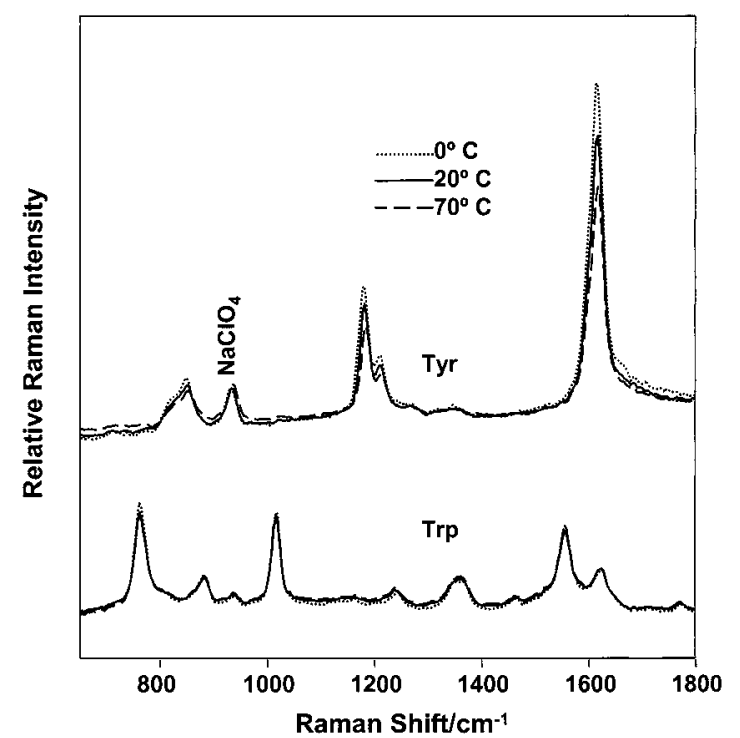

FIGURE 4: $229 \mathrm{~nm}$ excited UVRR of tyrosine in $0.20 \mathrm{M}$ sodium perchlorate at 0,20 , and $70{ }^{\circ} \mathrm{C} .229 \mathrm{~nm}$ excited UVRR of tryptophan in $0.20 \mathrm{M}$ sodium perchlorate at 0,20 , and $70{ }^{\circ} \mathrm{C}$.

chain, which signals a disruption of betanova's hydrophobic cluster. Thus, the UVRR data show that, although the $\beta$-sheet structure of betanova does not unfold significantly up to temperatures of $82{ }^{\circ} \mathrm{C}$, the Trp in its hydrophobic cluster becomes more water exposed. The inset to Figure 3 shows the dependence of the Trp peak intensity on temperature. The smoothly decreasing intensities show no evidence of a cooperative unfolding transition, for which we would expect a significantly sharper intensity dependence $(19,36)$.

The $229 \mathrm{~nm}$ excitation exclusively probes the aromatic side chains, while the $206.5 \mathrm{~nm}$ excitation permits independent probing of the backbone. Thus, we can conclusively state that, upon heating to $82^{\circ} \mathrm{C}$, the backbone conformation of betanova remains relatively unchanged, while the aromatic side chains show large spectral changes. These data indicate the presence of a collapsed intermediate state at elevated temperatures. This collapsed intermediate state has a nativelike backbone conformation with a disrupted hydrophobic cluster.

These interpretations conflict with those of Kortemme et al. (31), whose CD data indicated a decreasing ellipticity at $217 \mathrm{~nm}$ with increasing temperature. They assumed this ellipticity change resulted from unfolding of the $\beta$-sheet to a random coil structure. This assumption lead to their interpretation of a cooperative two-state thermal unfolding for betanova. In fact, the CD spectrum at $217 \mathrm{~nm}$ derives from the overlap of the Trp and Tyr CD $\pi \rightarrow \pi^{*}$ aromatic transitions and the peptide $\mathrm{n} \rightarrow \pi^{*}$ transitions. Thus, it is not possible to easily interpret these CD data, especially because of the weak ellipticity of the $\beta$-sheet backbone (37, 38). In contrast, our Raman data are able to distinguish that the spectral changes, which occur upon heating of the sample, derive exclusively from the aromatic groups. Additionally, we observe that the dependence of the CD data of Kortemme et al. on temperature has a gentle slope (31). If a cooperative two-state transition were occurring, we would anticipate this dependence to be more sigmoidal in shape $(19,35)$. Furthermore, in the case of a two-state $\beta$-sheet unfolding, we would predict the presence of an isodichroic point at $\sim 210 \mathrm{~nm}$ in the CD spectra (19). Since Kortemme and co- 


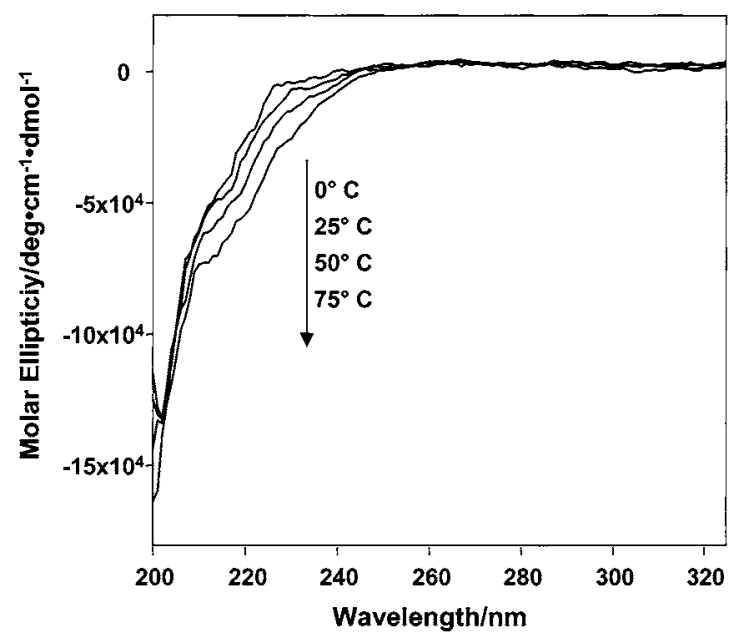

Figure 5: Temperature dependence of the betanova CD spectra. Solutions of $158 \mu \mathrm{M}$ betanova in $5 \mathrm{mM}$ sodium acetate buffer were measured at $0,25,50$, and $75^{\circ} \mathrm{C}$.

workers did not publish their actual CD spectra, we investigated this point by measuring the temperature dependence of the CD spectra (Figure 5). We do not observe the expected isodichroic point. Thus, we conclude that the observed $217 \mathrm{~nm}$ ellipticity decrease of Kortemme et al. (31) results primarily from changes in the aromatic ring $\mathrm{CD}$.

In contrast to the two-state model proposed by Kortemme et al. (31), Bursulaya and Brooks recently proposed an energy landscape that is likely to give rise to a three-state betanova unfolding mechanism (32). The folding of betanova was modeled using molecular dynamics to calculate the radius of gyration, the fraction of native hydrophobic contacts, and the potential of mean force. This resulted in a folding free energy landscape where three states (unfolded, collapsed, and native) were easily differentiated. Collapsed state conformers were differentiated from native and unfolded conformers in having a persistent native betanova secondary structure but only a fraction of the native hydrophobic contacts. The folding free energy landscape contains a broad energy minimum extending from the native state to the region of the collapsed state where $\sim 60 \%$ of the native hydrophobic contacts are present. These simulations predict a low-energy structure with a three-stranded $\beta$-sheet in the collapsed state where nearly $60 \%$ of the native hydrophobic contacts occur. Our UVRR results support Bursulaya and Brooks' threestate folding landscape (32), since we apparently detect their collapsed state.

There is significant controversy about the relative importance of the individual residue propensities, polar interactions, and hydrophobic interactions in the $\beta$-sheet folding mechanism $(14,16-19,32,39-41)$. The relative importance of these factors is likely to be peptide- and protein-specific. For the case of betanova, our results show that the hydophobic core loosens without impacting the secondary structure. Thus, our data suggest that hydrophobic packing interactions are not decisive in stabilizing betanova's $\beta$-sheet structure.

Both the NMR data presented by Kortemme et al. (31) and the molecular dynamics calculations of Bursulaya and Brooks (32) propose a folded state for betanova at low temperature. Additionally, Bursulaya and Brooks calculate a collapsed state for betanova at higher temperature. In view of these results, we have assumed a low-energy, folded state at $5{ }^{\circ} \mathrm{C}$ and have searched for a transition from this lowtemperature folded state to a collapsed, intermediate state at higher temperature.

Instead, we observe a continuous loosening of the hydrophobic cluster over a broad temperature range and no sharp secondary structure transition. In reality, we may be observing a structure with an average of $63 \% \beta$-sheet content over the entire temperature range. This would indicate the lack of a well-defined, folded structure. Thus, betanova's structure might best be described as a molten globule over the $3-82$ ${ }^{\circ} \mathrm{C}$ temperature range of our study.

\section{ACKNOWLEDGMENT}

We thank Dr. Zhenhuan Chi and Prof. John Rosenberg for helpful discussions.

\section{REFERENCES}

1. Burley, S. K., Almo, S. C., Bonanno, J. B., Capel, M., Chance, M. R., Gaasterland, T., Lin, D., Sali, A., Studier, F. W., and Swaminathan, S. (1999) Nat. Genet. 23, 151-157.

2. Service, R. F. (2000) Science 287, 1954-1956.

3. Kim, S.-H. (1998) Nat. Struct. Biol. 5, 643-645.

4. Botstein, D., and Cherry, J. M. (1997) Proc. Natl. Acad. Sci. U.S.A. 94, 5506-5507.

5. Fields, S., Kohara, Y., and Lockhart, D. J. (1999) Proc. Natl. Acad. Sci. U.S.A. 96, 8825-8826.

6. Dyson, H. J., and Wright, P. E. (1996) Annu. Rev. Phys. Chem. 47, 369-395.

7. Eaton, W. A., Munoz, V., Thompson, P. A., Henry, E. R., and Hofrichter, J. (1998) Acc. Chem. Res. 31, 745-753.

8. Gruebele, M. (1999) Annu. Rev. Phys. Chem. 50, 485-516.

9. Lednev, I. K., Karnoup, A. S., Sparrow, M. C., and Asher, S. A. (1999) J. Am. Chem. Soc. 121, 8074-8086.

10. Williams, S., Causgrove, T. P., Gilmanshin, R., Fang, K. S., Callender, R. H., Woodruff, W. H., and Dyer, R. B. (1996) Biochemistry 35, 691-697.

11. Harbury, P. B., Plecs, J. J., Tidor, B., Alber, T., and Kim, P. S. (1998) Science 282, 1462-1467.

12. Mayne, L., Englander, W., Qiu, R., Yang, J., Gong, Y., Spek, E. J., and Kallenbach, N. R. (1998) J. Am. Chem. Soc. 120, 10643-10645.

13. Bryson, J. W., Betz, S. F., Lu, H. S., Suich, D. J., Zhou, H. X., O’Neil, K. T., and DeGrado, W. F. (1995) Science 270, 935-941.

14. Blanco, F. J., Jimenez, A., Herranz, J., Rico, M., Santoro, J., and Nieto, J. L. (1993) J. Am. Chem. Soc. 115, 5887-5888.

15. (a) De Alba, E., Jiménez, M. A., and Rico, M. (1997) J. Am. Chem. Soc. 119, 175-183; (b) De Alba, E., Rico, M., and Jiménez, M. A. (1997) Protein Sci. 6, 2548-2560.

16. Haque, T. S., and Gellman, S. H. (1997) J. Am. Chem. Soc. 119, 2303-2304.

17. Griffiths-Jones, S. R., Maynard, A. J., and Searle, M. S. (1999) J. Mol. Biol. 292, 1051-1069.

18. Sieber, V., and Moe, G. R. (1996) Biochemistry 35, 181188.

19. (a) Sharman, G. J., and Searle, M. S. (1997) Chem. Commun. 20, 1955-1956; (b) Sharman, G. J., and Searle, M. S. (1998) J. Am. Chem. Soc. 120, 5291-5300.

20. Das, C., Raghothama, S., and Balaram, P. (1998) J. Am. Chem. Soc 120, 5812-5813.

21. Schenck, H. L., and Gellman, S. H. (1998) J. Am. Chem. Soc. 120, 4869-4870.

22. Doig, A. J. (1997) Chem. Commun. 22, 2153-2154.

23. Srinivasan, R., and Rose, G. (1995) Proteins: Struct., Funct., Genet. 22, 81-99.

24. Dill, K. A., and Chan, H. S. (1997) Nat. Struct. Biol. 4, 1019.

25. Chan, H. S., and Dill, K. A. (1998) Proteins: Struct., Funct., Genet. 30, 2-33. 
26. Socci, N. D., Onuchic, J. N., and Wolynes, P. G. (1996) J. Chem. Phys. 104, 5860-5868.

27. Nymeyer, H., Garcia, A. E., and Onuchic, J. N. (1998) Proc. Natl. Acad. Sci. U.S.A. 95, 5921-5928.

28. Doyle, R., Simons, K., Quian, H., and Baker, D. (1997) Proteins: Struct., Funct., Genet. 29, 282-291.

29. Ladurner, A. G., Itzhaki, L. S., Daggett, V., and Fersht, A. R. (1998) Proc. Natl. Acad. Sci. U.S.A. 95, 8473-8478.

30. Laurents, D. V., and Baldwin, R. L. (1998) Biophys. J. 75, $428-434$.

31. Kortemme, T., Ramirez-Alvarado, M., and Serrano, L. (1998) Science 281, 253-256.

32. Bursulaya, B. D., and Brooks, C. L., III (1999) J. Am. Chem. Soc. 121, 9947-9951.

33. (a) Holtz, J. S. W., Bormett, R. W., Chi, Z., Cho, N., Chen, X. G., Pajcini, V., Asher, S. A., Arrogoni, M., Owen, P., and Spinelli, L. (1996) Appl. Spectrosc. 50, 1459-1468; (b) Asher, S. A., Bormett, R. W., Chen, X. G., Lemmon, D. H., Cho, N., Peterson, P., Arrogoni, M., Spinelli, L., and Cannon, J. (1993) Appl. Spectrosc. 47, 628; (c) Asher, S. A., Johnson, C. R., and Murtaugh, J. (1983) J. Rev. Sci. Instrum. 54, 1657.
34. Chi, Z., Chen, X. G., Holtz, J. S. W., and Asher, S. A. (1998) Biochemistry 37, 2854-2864.

35. Chi, Z., and Asher, S. A. (1998) J. Phys. Chem. B 102, 95959602.

36. Bergethon, P. B. (1998) Physical Basis of Biochemistry. The Foundations of Molecular Biophysics, pp 381-385, Springer, New York.

37. Woody, R. W. (1996) in Circular Dichroism and the Conformational Analysis of Biomolecules (Fasman, G. D., Ed.) pp 25-67, Plenum Press, New York.

38. Woody, R. W., and Dunker, A. K. (1996) in Circular Dichroism and the Conformational Analysis of Biomolecules (Fasman, G. D., Ed.) pp 109-157, Plenum Press, New York.

39. (a) Li, H., Tang, C., and Wingreen, N. S. (1997) Phys. Rev Lett. 79, 765-768; (b) Wang, Z.-H., and Lee, H. C. (2000) Phys. Rev. Lett. 84, 574-577.

40. Wright, P. E., Dyson, H. J., and Lerner, R. A. (1988) Biochemistry 27, 7167-7175.

41. Minor, D. L., Jr., and Kim, P. S. (1994) Nature 367, 660-663.

BI011505K 